\title{
Lessons Learned from Right Ventricular Dilatation without Pulmonary Embolism on Contrast Enhanced Computed Tomography in Patients with Non-Severe COVID-19
}

\author{
Yuki Ishibashi $^{1}$, Nozomi Kotoku${ }^{1}$, Yuta Hagiwara ${ }^{2}$, Kazutaka Kakinuma ${ }^{3}$, \\ Yuta Nakamura ${ }^{4}$, Seido Ooka ${ }^{5}$, Hiroki Ikeda ${ }^{6}$, Tsutomu Sakurada ${ }^{7}$, \\ Yasuhiro Tanabe ${ }^{1}$, Masamichi Mineshita ${ }^{3}$, and Yoshihiro J. Akashi ${ }^{1}$
}

(Received for Publication: October 9, 2020)

\begin{abstract}
Purpose: The novel 2019 coronavirus disease (COVID-19) is causing the current epidemic of pneumonia in Japan. This report evaluates contrast-enhanced computed tomography (CECT) features affecting clinical worsening in patients with non-severe pneumonia.

Methods: Clinical data of 11 patients hospitalized at our institution due to non-severe COVID-19 pneumonia between April 1 and April 30, 2020, were analyzed retrospectively.

Results: One patient (9.1\%) deteriorated, and 10 patients (90.9\%) improved and/or stabilized. CECT was performed on three patients with D-dimer elevated to $>6$-fold above the upper limit of normal. No patients suffered pulmonary embolism (PE). Heparin treatment was administered for 3 patients with elevated D-dimer levels, and the one patient with the sign of a RV/LV ratio of $>1.0$ on CECT underwent intubation and respiratory management.

Conclusion: A sign of RV dilatation without PE on CECT may be useful for predicting rapid clinical worsening and appropriate management in patients with non-severe COVID-19 pneumonia.
\end{abstract}

Key words

Non-severe COVID-19 pneumonia, RV dilatation, contrast-enhanced computed tomography, D-dimer

\section{Introduction}

Since first appearing in Wuhan, Hubei Province, China, in December 2019, a novel coronavirus (COVID-19) has spread rapidly around the world ${ }^{1,2)}$. Most severe manifestations of COVID-19 cases, such as multiple organ failure and death, have been linked to markers of coagulation dysfunction, such as platelet reduction, and increases in prothrombin time and fibrin degradation products, specifically, D-dimer ${ }^{3,4)}$. Previous reports suggested that heparin treatment reduced the mortality of COVID-19 patients with elevated levels of D-dimer ${ }^{5}$. COVID-19 pneumonia has shown an association between increased D-dimer levels, disease progression and venous thrombosis ${ }^{5}$. However, as detection of COVID-19 pneumonia does not require the need for an intravenous contrast agent, patients with COVID-19 pneumonia are im-

1 Division of Cardiology, Department of Internal Medicine, St. Marianna University School of Medicine

2 Division of Neurology, Department of Internal Medicine, St. Marianna University School of Medicine

3 Division of Respiratory Medicine, Department of Internal Medicine, St. Marianna University School of Medicine

4 Division of Endocrinology and Metabolism, Department of Internal Medicine, St. Marianna University School of Medicine

5 Division of Rheumatology, Department of Internal Medicine, St. Marianna University School of Medicine

6 Division of Gastroenterology and Hepatology, Department of Internal Medicine, St. Marianna University School of Medicine

7 Division of Nephrology and Hypertension, Department of Internal Medicine St. Marianna University School of Medicine 
aged with non-contrast chest computed tomography (CT).

Therefore, the purpose of this study was to investigate the relation between contrast-enhanced computed tomography (CECT) and clinical features in patients with non-severe COVID-19 pneumonia.

\section{Materials and Methods}

\section{Study patients}

In total, 11 patients, who were hospitalized at our institution due to non-severe COVID-19 pneumonia between April 1 and April 30, 2020, were retrospectively investigated. Patients who required intubation and respiratory management were considered to have severe pneumonia. The study procedures were approved by the ethics committee at St. Marianna University School of Medicine, Japan (approval number: 4712).

\section{Eligibility criteria for contrast-enhanced com- puted tomography}

Non-contrast CT was performed in all patients as a baseline investigation on admission. CECT was performed for patients with D-dimer elevated to $>6$ fold above the upper limit of normal ${ }^{6,7)}$.

\section{Evaluation by CECT}

We assessed the images for the presence of pulmonary embolism (PE). Right ventricular (RV) and left ventricular (LV) parameters were measured by two experienced cardiologists who were blinded to the patients, as previously described ${ }^{8}$.

\section{Results}

Table 1 shows the patients' demographic information. Their mean age was 58.4 years, and $81.8 \%$ were male. The average body mass index (BMI) was $28.3 \mathrm{~kg} / \mathrm{m}^{2}$. One patient $(9.1 \%)$ deteriorated, and 10 patients $(90.9 \%)$ improved and/or stabilized.

Findings of the three patients with D-dimer levels exceeding $3.0 \mu \mathrm{g} / \mathrm{mL}$ ( $>6$-fold increase above the upper limit of normal) are summarized in Table 2. PE was not found in any of the patients. However, 13 days after the onset of COVID-19, one patient experienced deep venous thrombosis (case 1 in Table 2 and Figure 1). Fourteen days after the onset of COVID-19, one patient required intubation and respiratory management due to critical COVID-19, which seemed to be related to the sign of a $\mathrm{RV} / \mathrm{LV}$ ratio of $>1.0$ on CECT (case 1 in Table 2 and Figure 1). Conversely, patients with a RV/LV ratio $\leq 1.0$ on CECT did not suffer critical COVID-19. Fifteen days after the onset of COVID-19, one patient experienced paroxysmal atrial fibrillation (case 3 in Table 2 and Figure 1).

\section{Discussion}

The main findings of this report are as follows.: 1) Among the 11 patients hospitalized at our institution due to non-severe COVID-19 pneumonia, three patients had significantly elevated levels of D-dimer. 2) One patient required intubation and respiratory management. 3) The clinical severity of coronavirus infection, as observed in patients hospitalized due to

Table 1. Demographic and Clinical Characteristics of the 11 Patients

\begin{tabular}{cc}
\hline Characteristics & Value \\
\hline Age,ys & $58.4 \pm 13.8$ \\
Male & $9(81.8)$ \\
Body mass index, $\mathrm{kg} / \mathrm{m}^{2}$ & $28.3 \pm 7.7$ \\
Current smoker & $2(18.2)$ \\
Hypertention & $4(36.4)$ \\
Dyslipidemia & $2(18.2)$ \\
Diabetes mellitus & $1(9.1)$ \\
Hyperuricemia & $3(27.2)$ \\
Respiratory disease & $1(9.1)$ \\
Collagen disease & $1(9.1)$ \\
Sleep apnea syndrome & $1(9.1)$ \\
\hline
\end{tabular}

Values are mean $\pm \mathrm{SD}$ or $\mathrm{n}(\%)$.

Table 2. Case Summary of Patients with Elevation of D-dimer Exceeding $3.0 \mu \mathrm{g} / \mathrm{mL}$ ( $>6$ - fold rise over the upper limit of normal)

\begin{tabular}{|c|c|c|c|c|c|c|c|c|c|}
\hline Case No. & Age, yrs & Sex & $\begin{array}{l}\mathrm{BMI}, \\
\mathrm{kg} / \mathrm{m}^{2}\end{array}$ & Days from onset to admission & $\begin{array}{l}\text { D-dimer, } \mu \mathrm{g} / \mathrm{ml} \\
\text { (days from onset to } \\
\text { examination) }\end{array}$ & Days from onset to CECT & PE & DVT & New onset of atrial fibrillation \\
\hline 1 & 55 & Male & 31.7 & 9 & 6.5 (Day12) & Day 13 & No & Yes & No \\
\hline 2 & 62 & Male & 27.1 & 8 & 6 (Day14) & Day 16 & No & No & No \\
\hline 3 & 44 & Male & 33.5 & 10 & 4.5 (Day15) & Day 15 & No & No & Yes \\
\hline
\end{tabular}

BMI: body mass index; CECT: Contrast enhanced computed tomography; PE: pulmonary embolism; DVT: deep venous thrombosis 


\begin{tabular}{|c|c|c|c|}
\hline $\mathrm{RV}$ & $69.7 \mathrm{~mm}$ & $44.7 \mathrm{~mm}$ & $37.7 \mathrm{~mm}$ \\
\hline LV & $48.6 \mathrm{~mm}$ & $45.8 \mathrm{~mm}$ & $51.4 \mathrm{~mm}$ \\
\hline $\mathrm{RV} / \mathrm{LV}$ & 1.43 & 0.98 & 0.73 \\
\hline Type & RV dilatation & Normal RV & Normal RV \\
\hline
\end{tabular}

RV: right ventricle, $\mathrm{LV}$ : left ventricle

Figure 1. CECT measurements in the patients with elevation of D-dimer exceeding $3.0 \mu \mathrm{g} / \mathrm{mL}$ ( $>6$-fold above the upper limit of normal)

non-severe COVID-19 pneumonia, seemed to be related to the sign of a RV/LV ratio of $>1.0$ on CECT.

Alterations in hematological parameters in patients infected with COVID-19 have been reported in other studies. Tang et al. ${ }^{6,7)}$, in a series that included 183 patients with confirmed COVID-19 infection, found that patients with abnormal coagulation results, especially elevated fibrin degradation product levels such as D-dimer, had a higher risk of death. The occurrence of severe COVID-19 disease was related to the D-dimer levels in other studies as well ${ }^{6}$. Three patients in the present series also had significantly elevated levels of D-dimer.

CECT is currently used to confirm PE in most patients and can detect RV dilatation as well. In the present series, heparin treatment was administered for the three patients with D-dimer levels exceeding $3.0 \mu \mathrm{g} / \mathrm{mL}$ : however, only the one patient with the sign of a RV/LV ratio of $>1.0$ on CECT required intubation and respiratory management. RV dilatation is a symptom associated not only with acute PE but also with cardiopulmonary comorbidities. Unfortunately, transthoracic echocardiography was not performed on all patients; therefore, we cannot confirm this result. Ciceri et al. reported that, in predisposed individuals, alveolar viral damage is followed by an inflammatory reaction and by microvascular pulmonary thrombosis now termed microvascular COVID-19 lung vessels obstructive thromboinflammatory syndrome ${ }^{9}$. We hypothesize that microvascular pulmonary thrombosis might influence the elevation of D-dimer levels ${ }^{10)}$.
Recently, Li et al. reported that RV longitudinal strain (RVLS) was a powerful predictor of higher mortality in patients with COVID-1911). According to this report, the additional prognostic value of RVLS was substantially independent of LV systolic functional index, which failed to predict mortality in patients with COVID-1911). COVID-19 infection might cause both pulmonary and systemic inflammations, which may contribute to RV dilatation through RV overload and direct damage to cardiomyocytes ${ }^{11)}$. Current guideline advocates the use of non-contrast CT for the diagnosis, severity assessment, and monitoring of COVID-19 pneumonia ${ }^{12)}$. Although the mortality of non-severe COVID-19 pneumonia seems relatively low, patients with severe COVID-19 pneumonia are at high risk to be admitted to the intensive care unit (ICU). Therefore, accurate diagnosis and monitoring of disease progression from the early stages are important. If a sign of RV dilatation without $\mathrm{PE}$ is found on CECT in patients with non-severe COVID-19 pneumonia, they may need to be moved to the ICU in preparation for intubation and the use of extracorporeal membrane oxygenation.

\section{Limitations}

The sample size in this study was small and there was no control group. Therefore, the current findings need to be confirmed in a larger study.

\section{Conclusions}

A sign of RV dilatation without PE on CECT 
may be useful for predicting rapid clinical worsening and the need for appropriate management in patients with non-severe COVID-19 pneumonia.

\section{Conflicts of Interest}

Dr. Mineshita reports the receipt of grants and personal fees from Novartis Japan, Boehringer Ingelheim Japan, GSK, and AstraZeneca plc; grants from TAIHO Pharmaceutical Co., Kyowa Kirin Co., Pfizer Inc., SHIONOGI \& CO., LTD., Astellas Pharma Inc.; and personal fees from Bristol-Myers Squibb, outside the submitted work. None of the other authors have any conflicts of interest to declare.

\section{References}

1) Vernuccio F, Giambelluca D, Cannella R, et al. Radiographic and chest CT imaging presentation and follow-up of COVID-19 pneumonia: a multicenter experience from an endemic area. Emergency radiology 2020; 27: 623-632.

2) Wu C, Chen $X$, Cai Y, et al. Risk Factors Associated With Acute Respiratory Distress Syndrome and Death in Patients With Coronavirus Disease 2019 Pneumonia in Wuhan, China. JAMA internal medicine 2020; 180: 934-943.

3) Agarwal A, Pinho M, Raj K, et al. Neurological emergencies associated with COVID-19: stroke and beyond. Emergency radiology 2020; 27: 747-754.

4) Connors JM, Levy JH. COVID-19 and its implications for thrombosis and anticoagulation. Blood 2020; 135: 2033-2040.

5) Oudkerk M, Büller HR, Kuijpers D, et al. Diagnosis, Prevention, and Treatment of Thromboembolic Complications in COVID-19: Report of the National Institute for Public Health of the Netherlands. Radiology 2020; 297: E216-E222.

6) Tang N, Bai H, Chen X, et al. Anticoagulant treatment is associated with decreased mortality in severe coronavirus disease 2019 patients with coagulopathy. Journal of thrombosis and haemostasis 2020; 18: 1094-1099.

7) Tang N, Li D, Wang X, et al. Abnormal coagulation parameters are associated with poor prognosis in patients with novel coronavirus pneumonia. Journal of thrombosis and haemostasis 2020; 18: 844-847.

8) El-Menyar A, Nabir S, Ahmed N, et al. Diagnostic implications of computed tomography pulmonary angiography in patients with pulmonary embolism. Annals of thoracic medicine 2016; 11: 269-276.

9) Ciceri F, Beretta L, Scandroglio AM, et al. Microvascular COVID-19 lung vessels obstructive thromboinflammatory syndrome (MicroCLOTS): an atypical acute respiratory distress syndrome working hypothesis. Critical care and resuscitation 2020; 22: 95-97.

10) Magro C, Mulvey JJ, Berlin D, et al. Complement associated microvascular injury and thrombosis in the pathogenesis of severe COVID-19 infection: a report of five cases. Translational research : the journal of laboratory and clinical medicine 2020; 220: 1-13.

11) Li Y, Li H, Zhu S, et al. Prognostic Value of Right Ventricular Longitudinal Strain in Patients with COVID-19. JACC Cardiovascular Imaging 2020; 13: 2287-2299.

12) American Cpllege of Radiology. ACR Recommendations for the use of Chest Radiography and Computed Tomography (CT) for Suspected COVID-19 Infection. https://www.acr.org/Advo cacy-and-Economics/ACR-Position-Statements/ Recommendations-for-Chest-Radiography-andCT-for-Suspected-COVID19-Infection. Retrieved on April 26, 2020. 